OPEN ACCESS

Edited by:

Akio Adachi,

University of Tokushima, Japan

Reviewed by:

Dabing Zhang,

China Agricultural University, China

Wuxun Lu,

University of Nebraska-Lincoln, USA

*Correspondence:

Yin Li

muziyin08@163.com

Specialty section: This article was submitted to

Virology,

a section of the journa

Frontiers in Microbiology

Received: 29 December 2015 Accepted: 21 March 2016 Published: 31 March 2016

Citation:

Han K, Zhao D, Liu Y, Liu Q, Huang X,

Yang J, An F and Li Y (2016)

Quantitative Proteomic Analysis of Duck Ovarian Follicles Infected with

Duck Tembusu Virus by Label-Free LC-MS. Front. Microbiol. 7:463. doi: 10.3389/fmicb.2016.00463

\section{Quantitative Proteomic Analysis of Duck Ovarian Follicles Infected with Duck Tembusu Virus by Label-Free LC-MS}

\author{
Kaikai Han 1,2, Dongmin Zhao 1,2, Yuzhuo Liu ${ }^{1,2}$, Qingtao Liu ${ }^{1,2}$, Xinmei Huang 1,2, \\ Jing Yang ${ }^{1,2}$, Fengjiao $\mathrm{An}^{1,2}$ and $\mathrm{Yin}_{\mathrm{Li}}{ }^{1,2 *}$ \\ 1 Key Laboratory of Veterinary Biological Engineering and Technology, National Center for Engineering Research of Veterinary \\ Bio-products, Institute of Veterinary Medicine, Ministry of Agriculture, Jiangsu Academy of Agricultural Sciences, Nanjing, \\ China, ${ }^{2}$ Jiangsu Key Lab of Zoonosis, Jiangsu Co-Innovation Center for Prevention and Control of Important Animal \\ Infectious Diseases and Zoonoses, Yangzhou, China
}

Duck Tembusu virus (DTMUV) is a newly emerging pathogenic flavivirus that has caused massive economic losses to the duck industry in China. DTMUV infection mainly results in significant decreases in egg production in egg-laying ducks within 1-2 weeks post infection. However, information on the comparative protein expression of host tissues in response to DTMUV infection is limited. In the present study, the cellular protein response to DTMUV infection in duck ovarian follicles was analyzed using nanoflow high-performance liquid chromatography-electrospray tandem mass spectrometry. Quantitative proteomic analysis revealed 131 differentially expressed proteins, among which 53 were up regulated and 78 were down regulated. The identified proteins were involved in the regulation of essential processes such as cellular structure and integrity, RNA processing, protein biosynthesis and modification, vesicle transport, signal transduction, and mitochondrial pathway. Some selected proteins that were found to be regulated in DTMUV-infected tissues were screened by quantitative real-time PCR to examine their regulation at the transcriptional level, western blot analysis was used to validate the changes of some selected proteins on translational level. To our knowledge, this study is the first to analyze the proteomic changes in duck ovarian follicles following DTMUV infection. The protein-related information obtained in this study may be useful to understand the host response to DTMUV infection and the inherent mechanism of DTMUV replication and pathogenicity.

Keywords: proteomic analysis, duck, Tembusu virus, ovarian follicles, label-free LC-MS

\section{INTRODUCTION}

Since April 2010, a severe duck disease outbreak has emerged throughout the major duckproducing regions of eastern China. In addition to ducks, the disease has also affected geese, chickens, and sparrows. The infected ducks exhibited high fever, diarrhea, and other consistent signs including acute anorexia, antisocial behavior, rhinorrhea, ataxia, and paralysis (Su et al., 2011). Necropsy of the infected ducks consistently displayed severe ovarian hemorrhage, ovaritis, and regression. In addition, ruptured ovarian follicles and peritonitis were also found in some infected 
ducks, whereas enlarged spleen and leg muscle hemorrhage were occasionally noted. The infected egg-laying ducks presented a significant reduction in egg production from 20 to $60 \%$, and even up to $90 \%$ in some reported cases, within 1-2 weeks postinfection (Yu et al., 2013), thus causing a severe impact on poultry production and heavy economic losses in China.

Based on the clinical signs and pathological features, this disease was initially designated as duck hemorrhagic ovaritis (DHO). However, further laboratorial studies proved that the causative agent isolated from ducks and geese is Tembusu virus (TMUV), which is a member of the Ntaya virus (NTAV) group belonging to the family Flaviviridae, genus Flavivirus (Cao et al., 2011). Similar to other flaviviruses, TMUV is a single-stranded positive-sense RNA virus, with a genome of approximately 10.5 $\mathrm{kb}$ and a single open reading frame encoding a large polyprotein (Su et al., 2011). The polyprotein can be cleaved by viral and cellular proteases into three structural proteins (capsid [C]; membrane $[\mathrm{M}]$, and envelope $[\mathrm{E}]$ ) and seven nonstructural proteins (NS1, NS2a, NS2b, NS3, NS4a, NS4b, and NS5; Sun et al., 2014). Nevertheless, to date, information about host cell responses to DTMUV infection is limited.

To understand the pathogenesis of viral infection, knowledge about virus-virus and virus-host interactions is critical. A virus infection may produce dramatic effects on the host cell morphology, transcription and translation patterns, cytoskeleton, cell cycle, innate immune response of the host, and apoptosis pathways, and may also cause inflammation and alter stress responses (Zheng J. et al., 2011). As a result, many functional and morphological changes in the host cells are associated with significant changes in the patterns of expression of host cell genes. Therefore, information on proteome changes in the host following DTMUV infection may be crucial to understand the host response to the virus and viral pathogenesis. In recent times, comparative proteomic analysis has emerged as a valuable tool for the establishment of global host protein profile in response to virus infection. This technique has been used to investigate the proteome changes in cells infected in vitro with classical swine fever virus (Li et al., 2010), infectious bursal disease virus (Zheng et al., 2008), and porcine circovirus (Liu et al., 2014). In addition, this analysis has also been widely employed to examine the mechanisms of viral infection through comparative investigation of the proteome changes in the host tissue in response to infection in vivo, such as Marek's disease virus (Lu et al., 2010) and infectious bronchitis virus (Cao et al., 2012).

The DTMUV genome encodes 10 proteins that facilitate exploitation of the host cell machinery to complete the infection cycle and generate viral progeny. However, to the best of our knowledge, there are no reports on the changes in the host tissues in response to DTMUV infection. Thus, the knowledge on proteins involved in these cellular responses to DTMUV infection and the directional changes in protein expression is limited. Label-free proteomic analysis based on nano-flow high-performance liquid chromatography (HPLC)-electrospray tandem mass spectrometry (LC-MS/MS) is emerging as a powerful methodology to determine disease-specific targets. When compared with conventional proteomic technologies, the major advantage of this technique is sensitivity, with thousands of proteins directly identified in typical analyses. In addition, lowabundance proteins can be detected and quantitative information can be acquired from the spectral counts obtained for each peptide sequence (Kikuchi et al., 2012). In this study, we present the results of a label-free quantitative proteomic comparison of the proteome of DTMUV-infected versus mock-infected duck ovarian follicles. Analysis of the obtained data revealed major changes in the proteins involved in central cellular signaling, metabolic pathways, and immune responses. Some individual proteins that were found to be regulated were further validated using qRT-PCR and immunoblotting techniques.

\section{MATERIALS AND METHODS}

\section{Experimental Animals and Virus Infection}

Healthy 180-day-old shelducks were obtained from Siji Poultry Co., Ltd (Jintan, PR China). The ducks were maintained in special cages, and food and water were provided regularly. All animal experiments were conducted in accordance with the regulations and guidelines of animal experimentation outlined by the People's Government of Jiangsu Province. The ducks infected with Tembusu virus JS804 strain were maintained in our laboratory.

\section{Experimental Design}

A total of 24 180-day-old shelducks were randomly divided into two groups and housed in different rooms. One group $(n=12)$ was inoculated intranasally with $0.4 \mathrm{~mL} \times 10^{5.0} \mathrm{ELD}_{50} / \mathrm{mL}$ of the challenge virus, additionally, the other group $(n=12)$ was mockinfected with sterile PBS in the same manner. The two groups of ducks were housed separately in different rooms and observed daily for 10 days post inoculation (dpi) for disease symptoms. At 3, 5, 7, and $10 \mathrm{dpi}$, three ducks were randomly selected from each group and euthanized using $\mathrm{CO}_{2}$ inhalation. The whole ovarian follicles were rapidly separated and washed with ice-cold phosphate buffered saline (PBS). At necropsy, one portion of the ovarian follicles was collected, snap-frozen in liquid nitrogen, and maintained at $-80^{\circ} \mathrm{C}$ for subsequent use in two-dimensional gel electrophoresis and western blot analysis, whereas the other portion was utilized for RNA extraction using Axygen Total RNA extraction Kit (Axygen Biosciences, China).

\section{Protein Sample Preparation}

The ovarian follicles derived from DTMUV- and mock-infected ducks were washed thrice with ice-cold PBS and lysed using a lysis buffer containing $8 \mathrm{M}$ urea, $2 \mathrm{M}$ thiourea, 4\% CHAPS, and $30 \mathrm{mM}$ Tris- $\mathrm{HCl}$ at $18^{\circ} \mathrm{C}$ for $15 \mathrm{~min}$. Subsequently, the tissues and cells were disrupted by ultrasonication performed for 20 times with 5 s pulse on and 5 s pulse down at $30 \%$ amplitude. The homogenates obtained were centrifuged at $14,000 \mathrm{~g}$ and $4^{\circ} \mathrm{C}$ for $20 \mathrm{~min}$, and the supernatants were collected and stored in singleuse aliquots at $-80^{\circ} \mathrm{C}$ until use. The protein concentrations in the supernatants were determined using BCA assay (Sigma, USA).

\section{Gel-Assisted Digestion}

For in-gel digestion, $10 \mathrm{mM}$ dithiothreitol (DTT) was added to each sample and incubated for $1 \mathrm{~h}$ at $37^{\circ} \mathrm{C}$ to reduce the cysteine side chains. Then, $20 \mathrm{mM}$ iodoacetamide was added 
to the samples and incubated for $30 \mathrm{~min}$ in dark at room temperature to alkylate the cysteine side chains. The samples were subsequently diluted six fold with $25 \mathrm{mM}$ ammonium bicarbonate to reduce the urea concentration to $1 \mathrm{M}$, and then $2 \%(\mathrm{w} / \mathrm{w})$ modified trypsin (Sigma, USA) was added. The $\mathrm{pH}$ was adjusted to 8.0 with $250 \mathrm{mM}$ ammonium bicarbonate, and the samples were incubated for $16 \mathrm{~h}$ at $37^{\circ} \mathrm{C}$. The digestion efficiency was determined by LC-MS/MS of the digests (aliquots containing $1 \mu \mathrm{g}$ of the initial amount of protein) desalted by using C18 Cartridge (Sigma, USA) following manufacturer's instructions.

\section{LC-MS/MS}

The protein samples were analyzed by nano-flow highperformance liquid chromatography (HPLC)-electrospray tandem mass spectrometry (LC-MS/MS). The digests were separated by nano-flow liquid chromatography using a SC200 traps $150 \mu \mathrm{m} \times 100 \mathrm{~mm} \mathrm{RP}^{-C_{18}}$ Thermo EASY column (Thermo, USA) at a flow rate of $300 \mathrm{~nL} / \mathrm{min}$. The mobile phase A comprised $2 \%$ acetonitrile and $0.1 \%$ formic acid in water and mobile phase B consisted of $0.1 \%$ formic acid in $84 \%$ acetonitrile. Following equilibration of the column in $100 \%$ solvent $\mathrm{A}$, an aliquot of each digest $(10 \mu \mathrm{L}$ corresponding to $5 \mu \mathrm{g}$ of total protein) was injected. Subsequently, the organic content of the mobile phase was linearly increased to $45 \%$ over $100 \mathrm{~min}$, and then to $100 \%$ over $108 \mathrm{~min}$, maintained at $100 \%$ over $120 \mathrm{~min}$. The column effluent was directed to a nanospray ion source and attached to a hybrid linear ion trap-Orbitrap mass spectrometer (Thermo Scientific, USA). The peptides were analyzed in positive ion mode and information-dependent acquisition mode to automatically switch between MS and MS/MS acquisition. The MS data were obtained using a data-dependent top 10 method, which dynamically selected the most abundant precursor ions from the survey scan $(300-1800 \mathrm{~m} / \mathrm{z})$ for higher-energy collisional dissociation (HCD) fragmentation. The target value was determined based on predictive automatic gain control (pAGC), and the dynamic exclusion duration was $25 \mathrm{~s}$. Survey scans were acquired at a resolution of 70,000 at $\mathrm{m} / \mathrm{z} 200$, whereas the resolution for HCD spectra was set to 17,500 at $\mathrm{m} / \mathrm{z} 200$. The normalized collision energy was $30 \mathrm{eV}$ and the underfill ratio, which specifies the minimum percentage of the target value likely to be reached at maximum fill time, was defined as $0.1 \%$. The instrument was run with the peptide recognition mode enabled. The MS experiments were performed in triplicates for each sample.

\section{Data Analysis}

The MS data were analyzed using MaxQuant software (version 1.3.0.5). The peptides and related proteins were searched against the UniProt Anas platyrhynchos database (32,619 total entries, downloaded 02/09/15). The initial search was set at a precursor mass window of $6 \mathrm{ppm}$. The search followed an enzymatic cleavage rule of Trypsin/P and allowed a maximum of two missed cleavage sites and a mass tolerance of $20 \mathrm{ppm}$ for fragment ions. Carbamidomethylation of cysteines was defined as fixed modification, while protein $\mathrm{N}$-terminal acetylation and methionine oxidation were defined as variable modifications.
The cutoff of global false discovery rate (FDR) for peptide and protein identification was set to 0.01 . The identified peptides were subjected to intensity-based absolute quantification (iBAQ) in MaxQuant to quantify protein abundance. The protein abundance was calculated based on the normalized spectral protein intensity (LFQ intensity).

The sequence data of the selected differentially expressed proteins were retrieved in batches from UniProtKB database (Release 2015_06) in FASTA format. The retrieved sequences were locally searched against SwissProt database (duck) using the NCBI BLAST+ client software (ncbi-blast-2.2.28+-win32.exe) to find the homologous sequences from which the functional annotation could be assigned to the studied sequences. The top 10 blast hits with an $E$-value of less than 1e-3 for each query sequence were retrieved and loaded into Blast2GO2 (Version 2.8.0) for GO mapping and annotation. Following annotation and annotation augmentation steps, the studied proteins were also compared by BLAST against KEGG (Kyoto Encyclopedia of Genes and Genomes) GENES to retrieve their KOs and were subsequently mapped to pathways in KEGG.

\section{Western Blot Analysis}

To further verify the variation in the differentially expressed proteins identified by the proteomic approaches, 2-5-oligoadenylate synthase-like protein 2 (OASL2), interferoninduced protein with tetratricopeptide repeats 5 (IFIT5), histone deacetylase 2 (HDAC2), thrombospondin-1 (TPP1), and basement membrane-specific heparan sulfate proteoglycan (HSPG) were selected for western blot analysis. The analysis was performed as described previously (Chen et al., 2014) with minor modifications. Briefly, the protein samples (50 $\mu \mathrm{g} / \mathrm{lane})$ were separated using $12 \%$ SDS-PAGE gels and transferred to a nitrocellulose transfer membrane. After blocking with 5\% (w/v) skimmed milk in TBST (50 mM Tris, $\mathrm{pH} 8.0,150 \mathrm{mM}$ $\mathrm{NaCl}, 0.1 \%$ [v/v] Tween-20) for $1 \mathrm{~h}$ at $37^{\circ} \mathrm{C}$, the membranes were incubated overnight at $4{ }^{\circ} \mathrm{C}$ with primary rabbit polyclonal antibodies of anti-OASL2 (Abcam, Cambridge, UK), anti-IFIT5 (OriGene, China), anti-TPP1 (Santa Cruz Biotechnology, CA), anti-HSPG (Santa Cruz Biotechnology, CA), mouse polyclonal antibodies of anti-HDAC2 (Santa Cruz Biotechnology, CA) at a dilution of 1:1000. After washing with TBST three times, the membranes were further incubated for $1 \mathrm{~h}$ with horseradish peroxidase-conjugated goat anti-rabbit secondary antibody or goat anti-mouse secondary antibody (BOSTER, Wuhan, China) at a dilution of 1:5000. The immunoreactive protein bands were detected using 3,3'-diaminobenzidine (DAB; BOSTER, Wuhan, China), with $\beta$-actin as the loading control.

\section{Real-Time RT-PCR}

The total RNA was extracted from the ovarian follicles using Axygen Total RNA Extraction Kit (Axygen Biosciences, China) according to the manufacturer's instructions. Synthesis of cDNA was performed with $1 \mu \mathrm{g}$ of the extracted RNA using a RevertAid $^{\mathrm{TM}}$ First Strand cDNA Synthesis Kit (Fermentas, USA), according to the manufacturer's protocol. Specific primers for amplifying various target genes of the MS-identified proteins were designed according to the available gene sequences 
deposited in GenBank using Lasergene sequence analysis software (DNAStar, Inc., Madison, WI, USA; Table 2). Real-time RT-PCR was performed using SYBR Premix Ex $\mathrm{Taq}^{\mathrm{TM}}$ II Kit (Takara, China) on the LightCycler 480 real-time PCR system (Roche) as follows: $30 \mathrm{~s}$ at $95^{\circ} \mathrm{C}, 40$ cycles of denaturation at $95^{\circ} \mathrm{C}$ for $10 \mathrm{~s}$, and annealing and extension at $55^{\circ} \mathrm{C}$ for $45 \mathrm{~s}$. The melting curves were obtained and quantitative analysis of the data was performed using a relative quantification $\left(2^{-\Delta \Delta C T}\right)$ study model. The mock-infected ovarian follicles were used as control (relative expression $=1$ ) and $\beta$-actin was employed as an internal reference gene. Each sample was amplified in triplicate.

\section{RESULTS}

\section{DTMUV Infection and Clinical Signs}

From 3 to $6 \mathrm{dpi}$, transient depression and decreased food intake were observed in DTMUV-infected ducks. Furthermore, a lack of increase in body weight, greenish diarrhea, keratitis, and tearing were noted in the infected ducks. With the disease progression, some ducks exhibited abnormal gait and were unable to stand steadily and kept falling. Contrastingly, the mock-infected ducks remained healthy. The gross lesions of the sacrificed DTMUV-infected ducks mainly comprised severe ovarian hemorrhage, ovaritis, and regression consistently. In addition, ruptured ovarian follicles and peritonitis were also found. Although the viral RNAs were readily detected in the ovaries of the DTMUV-infected birds sacrificed on 3 and 5 dpi, they were not observed on 7 and 10 dpi (Table 1). In contrast, no viral RNAs were detected in the mock-infected group. These results indicated that the ducks in the DTMUVinfected group were successfully infected with Tembusu virus.

\section{Comparison of Differential Protein Expression in the Ovarian Follicles between DTMUV- and Mock-Infected Ducks}

Figure 1 shows the flowchart of the analysis of the ovarian follicles. For the identification of differentially expressed proteins in the ovarian follicles of the DTMUV-infected ducks, two sets of independent biological replicates of the ovarian follicles from the control (mock-infected group) and DTMUV-infected group were used. The lysates from the samples were used for gelassisted digestion and LC-MS/MS was performed in triplicate. A total of 1092 and 1176 unique peptides corresponding to 188 and 203 distinct proteins were identified from the two sets of biological replicates, respectively, by optimized LC-MS/MS analysis $(p<0.05$, protein score $\geq 34)$. Furthermore, 176 proteins from the two sets of biological replicates overlapped and were subsequently adjusted for multiple testing according to the stringent method of Benjamini and Hochberg (Cho et al., 2012).

In addition, the average coefficient of variation obtained for ratios of all proteins from two biological replicates (three analytical replicates) for both control and TMUV-infected ovarian follicles were determined to be $14 \%$ (corresponding to 1.2 -fold change). The cutoff criteria considered for the
TABLE 1 | Virus detection by an E-specific RT-PCR method in ducks infected by DTMUV.

\begin{tabular}{lc} 
Days post-infection & Virus detection in ovary* \\
\hline 3 & $3 / 3$ \\
5 & $3 / 3$ \\
7 & $0 / 3$ \\
10 & $0 / 3$
\end{tabular}

*Shown is the number of positive tissue from ducks/all three tissues from ducks tested in this timing.

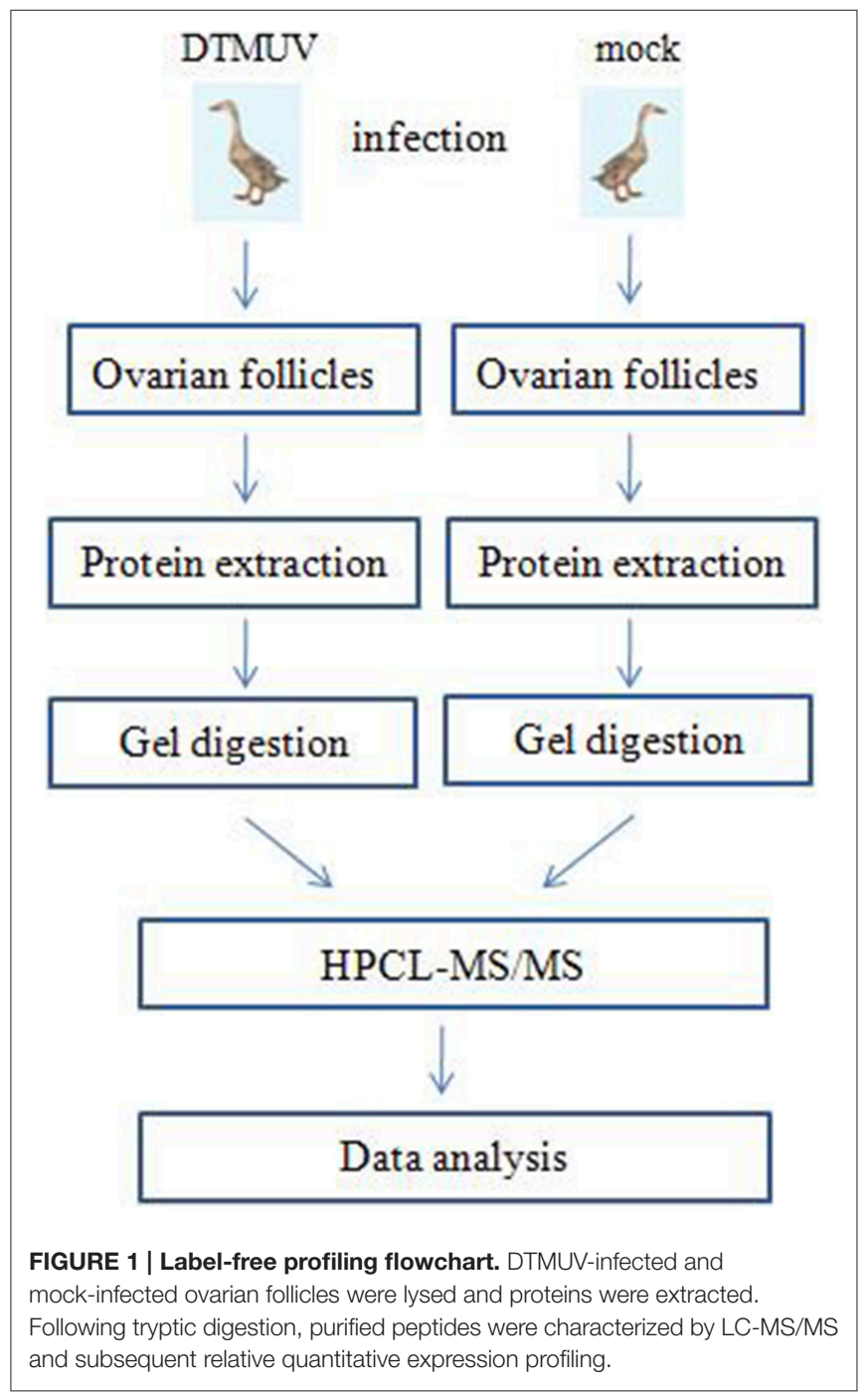

differentially expressed proteins were set with an adjusted $p$ value (Benjamini and Hochberg $(\mathrm{BH}) p$ value) of $<0.05$ and a ratio of $>2$-fold difference. Finally, 131 proteins among the quantified 176 proteins fulfilled the stringent cutoff criteria, of which 53 proteins were up regulated and 78 proteins were down regulated in the DTMUV-infected group. A list of partial regulated proteins is shown in Table 3. Table S1 (Supplemental Material) presents the entire list of proteins, whose expression was modified and 
TABLE 2 | Primers for qRT-CR analysis of 10 differentially expressed genes.

\begin{tabular}{|c|c|c|c|c|}
\hline Gene symbol & Gene accession NO. & Forward primer sequence $\left(5^{\prime}-3^{\prime}\right)$ & Reverse primer sequence $\left(5^{\prime}-3^{\prime}\right)$ & Amplicon size (bp) \\
\hline MGST3 & XM_005028171.2 & GAGCACGGGCGTATATTCAACTG & AGTGGCTAGGAAGAACAGGAAGG & 87 \\
\hline THBS1 & XM_005009791.2 & AGACACTGATTCTGACCGCATAGG & CTTGATTGGCATTGGGCACATAGG & 113 \\
\hline STX7 & XM_005009571.2 & GGTATCTGGTGGTGCTCCTGAAG & CTTGTGCTTGAGGTTGAGTGTCC & 77 \\
\hline NDVFA & XM_005029536.2 & CTGGAGGTGCCTGAGGTGAG & ACTGCGAGCCGTAGTGGTG & 70 \\
\hline TPP1 & XM_013108771.1 & CCTGGCTGCTGCTGCTC & GGCTGTCCTCCTCATCATCC & 81 \\
\hline IFIT5 & KF956064.1 & ACACTGTTGTTATGGCACTACTTG & AGCAGCATATCGCAGGAAATAAGG & 128 \\
\hline VLDL & ABJ16558.1 & GAGAGGGACCGTCGTGACTG & CATCCGCCAAGAACTGACCAAC & 106 \\
\hline HSPG & XM_013107098.1 & CCACCAACGCCTCCTTCCAG & GACAAATCCACCTTCTTCCСАTTG & 67 \\
\hline
\end{tabular}

the corresponding list of up and down regulated proteins. Protein ratios were presented as mock-infected/TMUV-infected.

The identified proteins were categorized according to the GO molecular functional groups. The main molecular functional groups of the proteins that exhibited significantly modified expression levels included catalytic proteins (28.8\%), binding proteins $(51.3 \%)$, transporter proteins $(6.41 \%)$, enzyme regulator proteins $(5.13 \%)$, electron carrier proteins $(1.28 \%)$, structural molecule proteins $(3.85 \%)$, channel regulator proteins $(0.64 \%)$, and molecular transducer proteins (0.64\%; Figure 2A). The cellular component of these identified proteins indicated that a majority of the shared proteins were localized in the cell (30.3\%), organelle $(24.8 \%)$, membrane $(13.2 \%)$, macromolecular complex (14.5\%), extracellular region (10.7\%), and extracellular matrix (2.99\%) (Figure S1). Furthermore, the identified proteins were found to be involved in diverse biological processes, such as cellular process, immune system process, biological regulation, signaling, metabolic process, biological adhesion, single-organism process, locomotion, response to stimulus, and multicellular organismal process (Figure 2B).

To better understand the interactions between the differentially expressed proteins during DTMUV replication and their possible involvement in viral pathogenesis, networks of inter-relationships of these proteins were constructed using the KEGG pathway program. A series of important pathways, including metabolism (e.g., citrate cycle, oxidative phosphorylation, terpenoid backbone biosynthesis), ECMreceptor interaction, focal adhesion, oxytocin signaling pathway, vitamin digestion, and absorption, and fat digestion and absorption was obtained (Figure 3). In addition, other wellknown pathways, including cell adhesion molecules (CAMs), PI3K-Akt signaling pathway, ubiquitin-mediated proteolysis, cAMP signaling pathway, and RIG-I-like receptor signaling pathway were also recorded.

\section{Analysis of the Identified Proteins at the Transcriptional Level}

Alterations in the expression of a protein may be owing to a change in its mRNA level. To confirm the results of the proteomic analysis at the mRNA level, the transcriptional alterations in 10 selected proteins were measured by real-time RT-PCR. The $\beta$-actin gene was used as the control housekeeping gene. In general, the trends in the change in mRNA abundance were similar to those of their corresponding proteins in the label-free LC-MS (Figure 4). The mRNA level of HSPG, MGST3, NDVFA, SDHB, THBS1, and VLVD were decreased in TMUV-infected ovarian follicles (Figure 4). The mRNA level of IFIT and IRF3 were increased in TMUV-infected ovarian follicles (Figure 4). There was no distinct change in the STX7 at the transcriptional level. This indicated that the fold change in protein expression of STX7 was mainly in the level of translation and post-translation modification. These data except STX7 provide transcriptional information complementary to those differentially expressed proteins detected by label-free LC-MS analysis.

\section{Confirmation of Proteomic Data by Western Blot Analysis}

To further verify the proteins identified by label-free LC-MS methods, five proteins (OASL2, IFIT5, HDAC2, TPP1, and HSPG) were selected for western blot analysis. Equal amounts of tissue lysates from TMUV-infected ovarian follicles and mockinfected ovarian follicles were examined with specific antibodies to these proteins. The data showed in Figure 5 indicated that these proteins were recognized with respective antibodies at different level. Also from Figure 5, it showed that IFIT5 and OASL2 were up regulated in TMUV-infected ovarian follicles, HDAC2, TPP1, and HSPG were down regulated in TMUVinfected ovarian follicles, these results was consistent with the label-free LC-MS analysis. All of above data validated the LCMS identification of those proteins in DTMUV-infected ovarian follicles that differentially expressed.

\section{DISCUSSION}

The egg-laying performance of layer ducks is the most important economic trait and is regarded as a critic factoring affecting productivity in the layer duck industry. Ovaries play an important role in laying trait. It has been reported that DTMUV affects both meat and laying ducks, significantly reducing egg production ranging from 20 to $60 \%$, and even up to $90 \%$, in the farms of eastern China (Li et al., 2015). At necropsy, the infected ducks displayed severe ovarian hemorrhage, ovaritis, 
TABLE 3 | List of differentially expressed proteins (53 up-regulated and 78 down-regulated) in TMUV-infected ovarian follicles versus mock-infected.

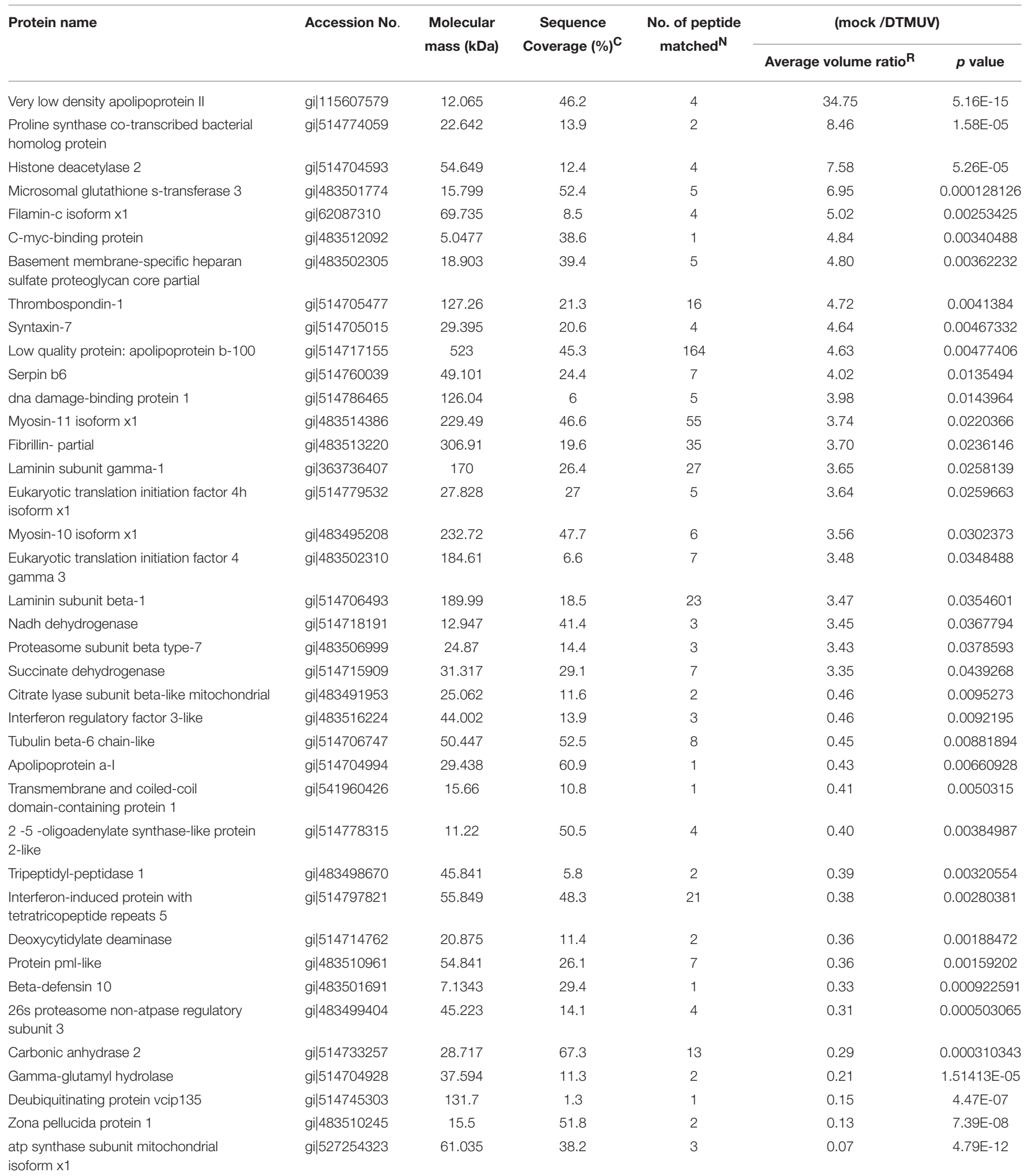

$R$, Protein expression ratio. Calculated by the mean of corresponding peptide intensities detected in mock-infected versus DTMUV-infected ovarian follicles; $N$, number of peptides detected; $C$, detected peptide coverage of protein sequence in \%; Values are means $\pm S E M s$. Compared with the control group, proteins with a threshold of $>2$ or, $<0.5$-fold and $P$ $<0.05$ were considered differentially expressed proteins. Proteins from two independent biological replicate sets from control and TMUV-infected were used for gel-assisted digestion and then triplicate $L C-M S / M S$ analysis. 

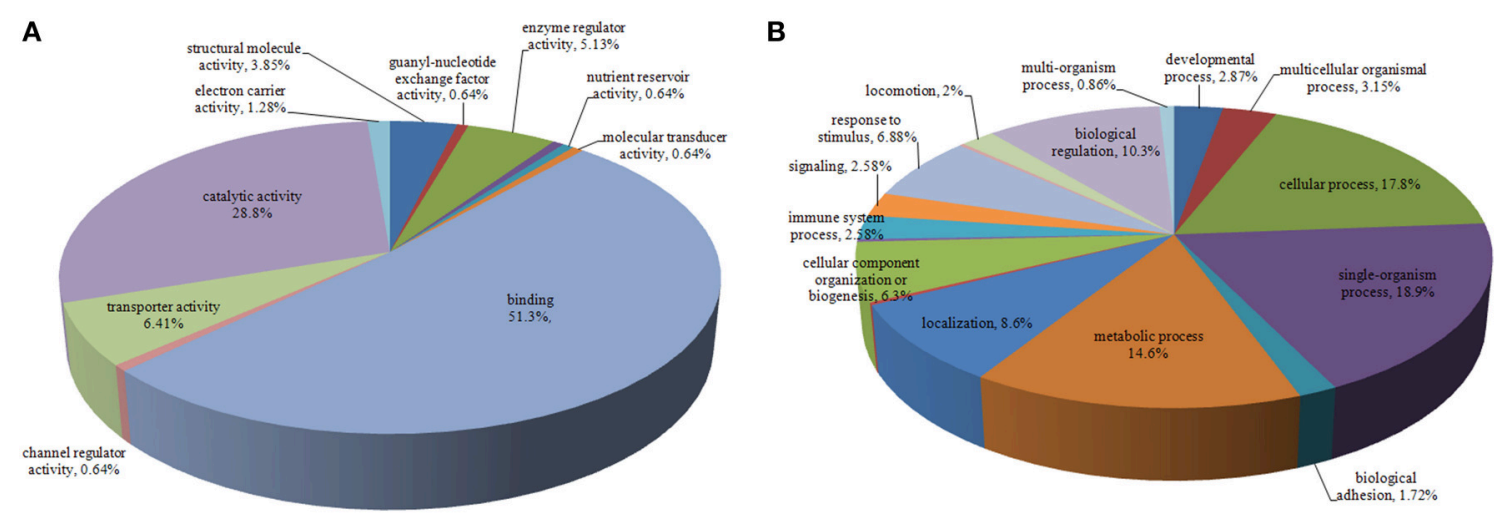

FIGURE 2 | Pie chart representing differentially expressed proteins identified by label-free LC-MS following DTMUV infected ovarian follicles. Proteins were classified according to their Molecular Function (A), Biological processes (B).

\begin{tabular}{|c|c|c|c|c|}
\hline $\begin{array}{r}\text { Citrate cycle (TCA cycle) } \\
\text { Oxidative phosphoryabion } \\
\text { Terpenoid backbone biosynthes is } \\
\text { ECM-receptor interacton } \\
\text { Focal adhesion } \\
\text { OxMocin signaling pathway } \\
\text { Vitamin diges son and absorpoon } \\
\text { Fat digesson and absorpton }\end{array}$ & $\begin{array}{l}2(2.74 \mathrm{e}-02) \\
2(1.23 \mathrm{e}-02) \\
\\
\\
2(1.59 \mathrm{e}-02) \\
2(3.57 \mathrm{e}-02) \\
\\
2(3.87 \mathrm{e}-02) \\
\end{array}$ & $\begin{array}{l}(6 e-04) \\
7(1.29 e-03)\end{array}$ & $\begin{array}{l}A A \\
A B \\
A B \\
C C \\
C D \\
E B \\
E D \\
E B\end{array}$ & $\begin{array}{l}\text { A } \\
\text { C } \\
\text { D } \\
\text { E }\end{array}$ \\
\hline & $4 \%$ & $10 \%$ & & \\
\hline & $\begin{array}{l}\text { Percen } \\
\text { A: Metabolism } \\
\text { C: Environmental Intormation Processing } \\
D: \text { Cellular Processes } \\
\text { E: Organismal Systems }\end{array}$ & $\begin{array}{l}\text { genes } \\
\text { AA : Carbohydrate metabolism } \\
\text { AB: Energymetabollsm } \\
\text { A : Metabolism of terpenoids and polyketdes } \\
\text { CC: Signaling molecules and interacton } \\
D D \text { : Celluluar community } \\
\text { EB: Endocrine system } \\
\text { ED : Digestive system }\end{array}$ & & \\
\hline
\end{tabular}

and regression consistently. DTMUV infection may cause dramatic changes in the function and morphology of the host tissues, and even alter the expression of the host cell genes. Recently, increasing attentions have been focused on the interaction between flavivirus and host (Zheng J. et al., 2011). However, there are none studies that have been performed to analyze this interaction using proteomics methods.

Two-dimensional LC-MS/MS enables identification of proteins in a complex mixture by using a combination of HPLC and MS after tryptic proteolysis, and is a powerful method for investigating disease biomarkers (Bartel et al., 2011). In the present study, two-dimensional LC-MS/MS proteomics was used to compare the total proteomes in the DTMUV-infected ovarian follicles with those in the normal ovarian follicle tissues. Quantitative analysis demonstrated dysregulation of 131 proteins in the DTMUV-infected ovarian follicles (53 up regulated and 78 down regulated proteins). The differentially expressed proteins were found to be involved in cytoskeleton organization, macromolecular biosynthesis, signal transduction, stress response, ubiquitin-proteasome pathway (UPP), and metabolic enzymes. All of these results indicate that the development of DTMUV in ovarian follicles is a process that involves multiple factors, in which many proteins and pathways are involved and where binding functions are greatly affected by pathogenesis.

\section{Changes in Host Cytoskeleton after DTMUV Infection}

The cytoskeletal network is a cellular scaffold system whose functions include maintenance of cellular shape, enabling cellular migration, cell division, intracellular transport, signaling, and membrane organization. Many viruses, such as herpes viruses (Falke, 1997), human immunodeficiency virus (HIV; Fackler and Krausslich, 2006), mammalian reovirus (Parker et al., 2002), etc. utilize the host cytoskeleton to promote infection. In the present study, $\beta$-tubulin, apolipoprotein, transmembrane, and coiled-coil domain-containing protein were up regulated, whereas filaminc isoform, fibrillin, myosin-10 isoform, myosin-11 isoform, and unconventional myosin-ic were down regulated in the DTMUVinfected group.

$\beta$-tubulin is a microtubule-associated protein, which plays important roles in the lifecycle of viruses. Changes in $\beta$-tubulin 
have been detected in SARS-CoV22 (Jiang et al., 2005) and infectious bursal disease virus (Zheng et al., 2008). Fan et al. (2012) indicated that the $\beta$-tubulin level was down regulated in infected PK-15 cells, and speculated that the $\beta$-tubulin networks collapse and disperse in host cells leading to an unstable cytoskeletal structure and release of viral particles from the infected cells. Myosins are a large superfamily of motor proteins that are involved in movement along actin filaments, development of myriad cells, targeted organelle transport, endocytosis, chemotaxis, cytokinesis, and signal transduction (Li and Yang, 2015). Some viral proteins can interact with the cytoskeletal transport machinery, such as actin-binding proteins, and induce rearrangements of cytoskeletal filaments so that they

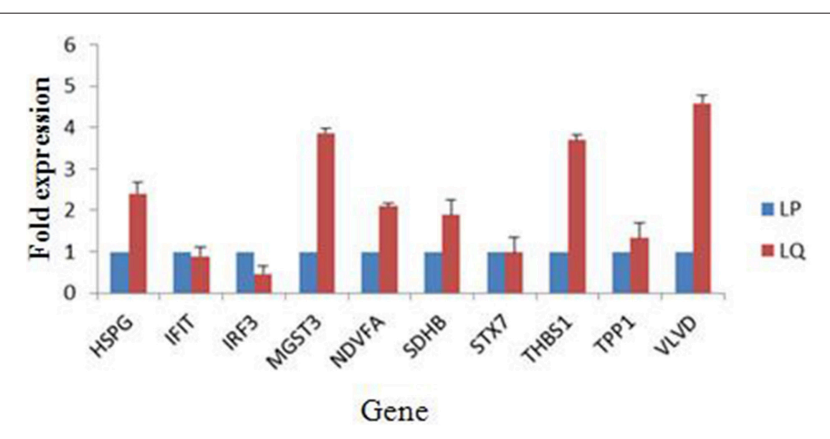

FIGURE 4 | Transcript alteration of the differentially expressed proteins in DTMUV-infected ovarian follicles. Total RNA of ovarian follicles (LP, DTMUV- infection; LQ, mock-infection) was measured by real time RT-PCR analysis. Samples were normalized with $\beta$-actin gene as a control housekeeping gene. Error bars represent standard deviation. Gene symbols indicating different genes listed as follows. HSPG: heparan sulfate proteoglycan; IFIT, interferon-induced protein with tetratricopeptide repeats; IRF3, interferon regulatory factor 3-like protein; MGST3, microsomal glutathione s-transferase 3; NDVFA, NADH dehydrogenase; SDHB, succinate dehydrogenase; STX7, syntaxin-7; THBS1, thrombospondin-1; TPP1, tripeptidyl-peptidase 1; VLVD, very low density apolipoprotein. can utilize them as tracks or push them aside when they represent barriers (Radtke et al., 2006). In the present study, several actin-binding proteins, such as myosin-10 isoform, were found to be down regulated in the DTMUV-infected group, suggesting that DTMUV may also manipulate the host cytoskeletal network for its own infectious processes and replication. Nevertheless, the roles of these cytoskeletal proteins in ducks after DTMUV infection should be further investigated.

\section{Energy Production and Metabolism}

Viral replication requires energy and macromolecular precursors derived from the metabolic network of the host. In the present study, the abundance of some proteins associated with carbohydrate, amino acid, and lipid metabolic processes was found to be differentially altered in the DTMUV-infected group. In addition, we identified changes in the abundance of energy metabolism proteins, including $\beta$-citrate lyase, deoxycytidylate deaminase, mitochondrial ATP synthase and $\gamma$ glutamyl hydrolase, which were up regulated following DTMUV infection. Moreover, down regulation of enzymes, such as proline synthase, serpin b6, NADH dehydrogenase, and succinate dehydrogenase was also observed in the DTMUV-infected group. Mitochondrial ATP synthase catalyzes ATP synthesis using an electrochemical gradient of protons across the inner membrane during oxidative phosphorylation (Devenish et al., 2000). It is constitutively expressed in the inner mitochondrial membrane in normal cells and is involved in the regulation of a variety of cellular functions (Maguire et al., 2006). Abnormal expression of ATP synthase is found to be involved in cell dysfunction and tumorigenesis (López-Ríos et al., 2007). Recently, ATP synthase was reported as a proviral factor that can promote replication of herpes simplex virus-1 (Zheng S. et al., 2011) and H3N2 swine influenza virus (Wu et al., 2013). Thus, the increased ATP synthase expression observed in the present study may possibly contribute to virus replication. ATP citrate lyase catalyzes the generation of acetyl-CoA, a pivotal precursor of fatty acids, from
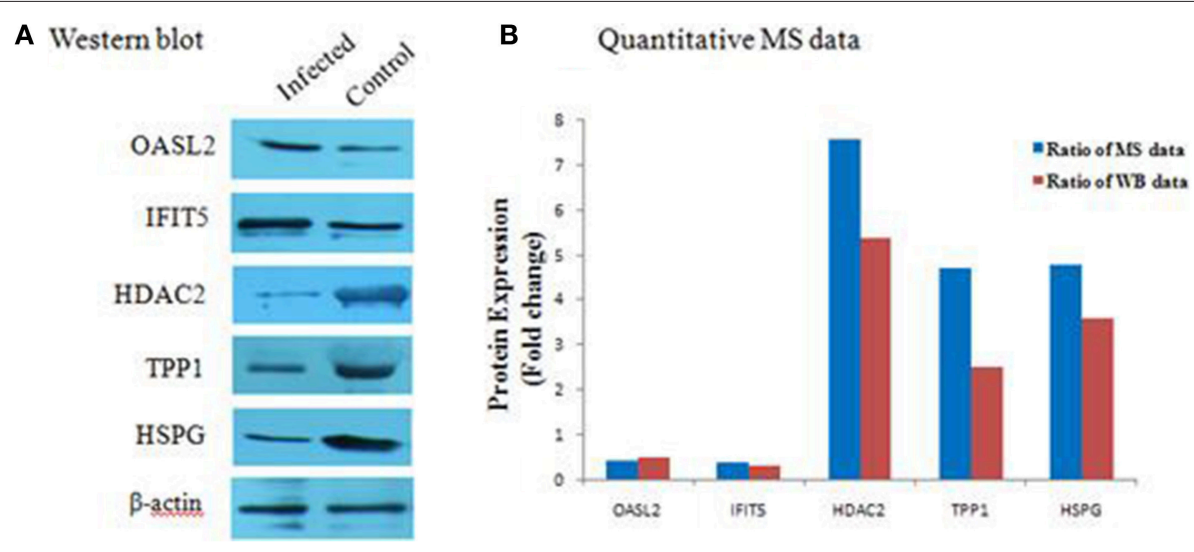

FIGURE 5 | Western blot analysis of representative proteins identified by LCMS/MS. (A) differential expression level of proteins, including OASL2, IFIT5, HDAC2, TPP1, and HSPG were detected by western blot analysis. After tissue lysis, equal amount of protein sample (50 $\mu \mathrm{g} / \mathrm{lane})$ were separated by SDS-PAGE. Western blot analysis was then performed using antibodies to these proteins. The $\beta$-actin was used as an internal control to normalize the quantitative data. (B) results derived from western blot analysis were compared with those from MS analysis. 
the accumulated citrate in the cytoplasm (Qian et al., 2015). In the flavivirus Hepatitis $\mathrm{C}$ virus ( $\mathrm{HCV}$ ), this enzyme is required for cholesterol and fatty acid biosynthesis, and is induced during the initial stages of high-level HCV replication. In addition, more considerable evidence suggests that the cholesterol and fattyacid-biosynthesis pathways may play a role in $\mathrm{HCV}$ replication and infection (Kapadia and Chisari, 2005). Meanwhile, the down-regulation of proteins involved in the glycolytic pathway and TCA cycle suggests that DTMUV infection reduces energy metabolism of the infected cells. This finding is consistent with some previous reports (Fan et al., 2012; Pando-Robles et al., 2014), demonstrating that DTMUV infection promotes changes in mitochondrial bioenergetics, causing an increase in cellular oxygen consumption and decreased efficiency in ATP synthesis (El-Bacha et al., 2007). However, the functional implications of these changes have not been demonstrated and need further research.

\section{RNA Processing and Translation Machinery}

Viruses may inhibit host protein synthesis by targeting multiple steps in the gene expression process via various pathways. For example, the vesicular stomatitis virus $M$ protein inhibits the initiation of the transcription of host genes (Ahmed et al., 2003) and the SARS-CoV spike protein inhibits host cell translation by interaction with eIF3f (Xiao et al., 2008). In the present study, we noted altered expression of several proteins following DTMUV infection, including down-regulation of two eukaryotic translation initiation factors (eIF4 and eIF4H) and c-mycbinding protein and up-regulation of $26 \mathrm{~s}$ proteasome. The protein eIF4H stimulates protein biosynthesis, ATP hydrolysis, and helicase activity of eIF4A (Bartel et al., 2011). The eukaryotic initiation factor 4 gamma 3 (eIF4G3) is critical for the initiation of protein translation. The eIF4G3 protein is a central player in the initiation of translation since it also contains binding domains for mRNA, eIF4A, and eIF3. It has been reported that many viruses cleave eIF4G3 to stop host protein translation (Marcet-Palacios et al., 2011). For example, rhinoviruses and enteroviruses shut off host protein synthesis by cleaving eIF4G3, resulting in inhibition of cap-dependent translation (Svitkin et al., 1999). Likewise, DTMUV may also play a similar role during its multiplication in host cells.

\section{The Alteration of Ubiquitin-Proteasome Pathway (UPP)}

UPP, a major intracellular protein degradation pathway, has recently been implicated in viral infections, including avoidance of host immune surveillance, viral maturation, viral progeny release, efficient viral replication, and reactivation of the virus from latency (Gao and Luo, 2006). In the present study, DTMUV infection induced differential expression of the deubiquitinating protein VCIP135, proteasome 26S subunit, and proteasome subunit beta. It has been demonstrated that VCIP135 possesses deubiquitinating activity, which is essential for p97/p47mediated Golgi membrane fusion (Zhang and Wang, 2015). The induced levels of VCIP135 observed in the present study might represent a cellular response to counterbalance cellular ubiquitination and degradation, which may facilitate viral progeny release. Nevertheless, further investigations are necessary to determine whether DTMUV possesses another strategy to establish infection.

\section{Proteins Associated with Immune Response}

The abundance of several proteins involved in immune response as well as antigen processing and presentation were also altered following DTMUV infection. The IFIT protein family, comprising IFIT1, IFIT2, IFIT3, and IFIT5, is characterized by multiple repeats of tetratricopeptide repeat helix-turn-helix motifs that mediate a variety of protein-protein interactions (Zhang et al., 2013). These proteins are distinctly induced by type I and II interferons and viruses, which are less abundantly expressed under normal conditions and are mainly concentrated in actin-rich protrusions and apical cell surfaces (Hsu et al., 2013). The IFITs are conserved in mammals, amphibians and fishes, but do not exist in lower animals. In birds, only IFIT5 has been detected (Zhou et al., 2013), and knowledge on its function is very limited. Recently, some reports have indicated that IFIT5 enhances NF- $\kappa \mathrm{B}$ activation through increased phosphorylation and activation of I $\kappa$ B kinase (IKK; Yamamoto and Gaynor, 2004). IKK catalyzes stimulus-induced phosphorylation and ubiquitinmediated degradation of $\mathrm{I} \kappa \mathrm{B}$ proteins, subsequently activating NF- $\kappa$ B. Further research has suggested that IFIT5 interacts with both IKK and TGF- $\beta$-activated kinase 1 (TAK1; Fan et al., 2011). Moreover, IFIT5 facilitates the interaction between TAK1 and IKK that contributes to IKK phosphorylation. Zhang et al. (2013) reported that IFIT5 was significantly induced at both mRNA and protein levels upon Sendai virus infection, and that the host antiviral responses were markedly enhanced or impaired in the presence or absence of IFIT5, respectively. In the present study, we detected up-regulation of IFIT5 following DTMUV infection in the ovarian follicles, however, its role in antiviral process and immune regulation requires further investigation.

The 2'-5' oligoadenylate synthase (OAS) family consists of OAS1, OAS2, OAS3, and OAS-like protein (OASL). The oligomerized OAS protein generates $2^{\prime}-5^{\prime}$-linked oligoadenylate (2-5A), activating endoribonuclease and RNase L (Pulit-Penaloza et al., 2012). It has been demonstrated that RNase L contributes to the control of early spread of virus by degrading the viral RNA and activating cytoplasmic pattern recognition receptors, including RIG-I and MDA-5 (Zhu et al., 2014). Recently, the OAS family proteins were noted to show RNase-L-dependent antiviral activity against dengue virus, West Nile virus, HCV, and Japanese encephalitis virus (Deo et al., 2014), all of which belong to the family Flaviviridae. Thus, the OASL screened in the present study may be associated with differential susceptibility to the clinical outcomes of Tembusu virus infection.

\section{CONCLUSIONS}

In summary, we have performed the first analysis of the proteomic changes in duck ovarian follicles during DTMUV infection in vivo. Also, we have shown that infection of ovarian follicles induces changes in expression of 131 proteins. Our 
data revealed significant changes in the proteins associated with energy metabolism, RNA processing and translation machinery, host cytoskeleton, and immune response. The modulation of protein expression found in our study may attribute to the strategy of the virus to overcome host pathways to facilitate survival at the expense of the host. Further, studies are needed to understand the detailed mechanism by which these proteins are induced during viral infection. Virus could make use of the transcription and translation of some host products for their survival, but the detailed mechanism is still unknown. The modulation of protein expression could also be the host's response to the viral infection. It would be interesting in a further study, to determine whether a similar response could occur in cells infected with other viruses or if the observed modulation of protein expression is specifically induce in response to DTMUV infection. Improvements in our knowledge on target organ will be important to decipher the infection process of Tembusu virus in ducks.

\section{REFERENCES}

Ahmed, M., McKenzie, M., Puckett, S., Hojnacki, M., Poliquin, L., and Lyles, D. (2003). Ability of the matrix protein of vesicular stomatitis virus to suppress beta interferon gene expression is genetically correlated with the inhibition of host RNA and protein synthesis. J. Virol. 77, 4646-4657. doi: 10.1128/JVI.77.8.4646-4657.2003

Bartel, S., Doellinger, J., Darsow, K., Bourquain, D., Buchholz, R., Nitsche, A., et al. (2011). Proteome analysis of vaccinia virus IHD-Winfected HEK 293 cells with 2-dimensional gel electrophoresis and MALDI-PSD-TOF MS of on solid phase support N-terminally sulfonated peptides. Virol. J. 8, 380. doi: 10.1186/1743-422X-8-380

Cao, Z., Han, Z., Shao, Y., Liu, X., Sun, J., Yu, D., et al. (2012). Proteomics analysis of differentially expressed proteins in chicken trachea and kidney after infection with the highly virulent and attenuated coronavirus infectious bronchitis virus in vivo. Proteome Sci. 10, 24. doi: 10.1186/1477-5956-10-24

Cao, Z., Zhang, C., Liu, Y., Liu, Y., Ye, W., Han, J., et al. (2011). Tembusu virus in ducks, china. Emerg. Infect. Dis. 17, 1873-1875. doi: 10.3201/eid1710.101890

Chen, W., Wu, Y., Chen, T., Cheng, C., Chan, H., Chou, H., et al. (2014). Proteomics analysis of the DF-1 chicken fibroblasts infected with avian reovirus strain S1133. PLoS ONE 9: e92154. doi: 10.1371/journal.pone.0092154

Cho, Y., Singh, T., Lee, H., Moon, P., Lee, J., Lee, M., et al. (2012). In-depth identification of pathways related to cisplatin-induced hepatotoxicity through an integrative method based on an informatics-assisted label-free protein quantitation and microarray gene expression approach. Mol. Cell. Proteomics 11: M111.010884. doi: 10.1074/mcp.M111.010884

Deo, S., Patel, T., Dzananovic, E., Booy, E., Zeid, K., McElency, K., et al. (2014). Activation of $2^{\prime} 5^{\prime}$-oligoadenylate synthetase by stem loops at the $5^{\prime}$-end of the West Nile Virus Genome. PLoS ONE 9:e92545. doi: 10.1371/journal.pone.0092545

Devenish, R., Prescott, M., Roucou, X., and Nagley, P. (2000). Insights into ATP synthase assembly and function through the molecular genetic manipulation of subunits of the yeast mitochondrial enzyme complex. Biochim. Biophys. Acta 1458, 428-442. doi: 10.1016/s0005-2728(00)00092-x

El-Bacha, T., Midlej, V., Pereira da Silva, A., Silva da Costa, L., Benchimol, M., Galina, A., et al. (2007). Mitochondrial and bioenergetic dysfunction in human hepatic cells infected with dengue 2 virus. Biochim. Biophys. Acta 1772, 1158-1166. doi: 10.1016/j.bbadis.2007.08.003

Fackler, O., and Krausslich, H. G. (2006). Interactions of human retroviruses with the host cell cytoskeleton. Curr. Opin. Microbiol. 9, 409-415. doi: 10.1016/j.mib.2006.06.010

\section{AUTHOR CONTRIBUTIONS}

$\mathrm{KH}$ and YL designed experiments; $\mathrm{KH}, \mathrm{DZ}, \mathrm{YL}, \mathrm{QL}$, and $\mathrm{XH}$ carried out experiments; JY and FA analyzed experimental results; $\mathrm{KH}$ wrote the manuscript.

\section{ACKNOWLEDGMENTS}

This work was supported by National Natural Science Foundation of China (No. 31502101), the Jiangsu Natural Sciences Foundation (BK20130710), Jiangsu Provincial Agricultural Science and Technology Innovation Foundation [No. cx (12) 5048].

\section{SUPPLEMENTARY MATERIAL}

The Supplementary Material for this article can be found online at: http://journal.frontiersin.org/article/10.3389/fmicb. 2016.00463

Falke, D. (1997). Herpes simplex virus and the cytoskeleton. Trends Microbiol. 5, 306. doi: 10.1016/S0966-842X(97)82226-3

Fan, H., Ye, Y., Luo, Y., Tong, T., Yan, G., and Liao, M. (2012). Quantitative proteomics using stable isotope labeling with amino acids in cell culture reveals protein and pathway regulation in porcine circovirus type 2 infected PK-15 cells. J. Proteome Res. 11, 995-1008. doi: 10.1021/pr200755d

Fan, Y., Yu, Y., Mao, R., Tan, X., Xu, G., Zhang, et al. (2011). USP4 targets TAK1 to downregulate TNFalpha-induced NF-kappaB activation. Cell Death Differ. 18, 1547-1560. doi: 10.1038/cdd.2011.11

Gao, G., and Luo, H. (2006). The ubiquitin-proteasome pathway in viral infections. Can. J. Physiol. Pharmacol. 84, 5-14. doi: 10.1139/y05-144

Hsu, Y., Shi, S., Wu, W., Ho, L., and Lai, J. (2013). Protective roles of interferon-induced protein with tetratricopeptide repeats 3 (IFIT3) in dengue virus infection of human lung epithelial cells. PLOS ONE 8: e79518. doi: 10.1371/journal.pone.0079518

Jiang, X., Tang, L., Dai, J., Zhou, H., Li, S., Xia, Q., et al. (2005). Quantitative analysis of Severe Acute Respiratory Syndrome (SARS)-associated coronavirusinfected cells using proteomic approaches. Mol. Cell Proteomics 4, 902-913. doi: 10.1074/mcp.M400112-MCP200

Kapadia, S., and Chisari, F. (2005). Hepatitis C virus RNA replication is regulated by host geranylgeranylation and fatty acids. Proc. Natl. Acad. Sci. U.S.A. 102, 2561-2566. doi: 10.1073.pnas.0409834102

Kikuchi, T., Hassanein, M., Amann, J. M., Liu, Q., Slebos, R. J., Rahman, S. M., et al. (2012). In-depth proteomic analysis of nonsmall cell lung cancer to discover molecular targets and candidate biomarkers. Mol. Cell Proteomics 11, 916-932. doi: 10.1074/mcp.M111.015370

Li, N., Wang, Y., Li, R., Liu, J., Zhang, J., Cai, Y., et al. (2015). Immune responses of ducks infected with duck Tembusu virus. Front. Microbiol. 6:425. doi: 10.3389/fmicb.2015.00425

Li, S., Qu, H., Hao, J., Sun, J., Guo, H., Guo, C., et al. (2010). Proteomic analysis of primary porcine endothelial cells after infection by classical swine fever virus. Biochim. Biophys. Acta 1804, 1882-1888. doi: 10.1016/j.bbapap.2010. 05.011

Li, Y., and Yang, W. (2015). Myosin superfamily: The multi-functional and irreplaceable factors in spermatogenesis and testicular tumors. Gene 576, 195-207. doi: 10.1016/j.gene.2015.10.022

Liu, J., Bai, J., Zhang, L., Hou, C., Li, Y., and Jiang, P. (2014). Proteomic alteration of PK-15 cells after infection by porcine circovirus type 2. Virus Genes 49, 400-416. doi: 10.1007/s11262-014-1106-4

López-Ríos, F., Sánchez-Aragó, M., García-García, E., Ortega, A., Berrendero, J., Pozo-Rodríguez, F., et al. (2007). Loss of the mitochondrial bioenergetic 
capacity underlies the glucose avidity of carcinomas. Cancer Res. 67, 9013-9017. doi: 10.1158/0008-5472.CAN-07-1678

Lu, Z., Qin, A., Qian, K., Chen, X., Jin, W., Zhu, Y., et al. (2010). Proteomic analysis of the host response in the bursa of Fabricius of chickens infected with Marek's disease virus. Virus Res. 153, 250-257. doi: 10.1016/j.virusres.2010.08.010

Maguire, D., Shah, J., and McCabe, M. (2006). Assaying ATP synthase rotor activity. Adv. Exp. Med. Biol. 578, 67-72. doi: 10.1007/0-387-29540-2_11

Marcet-Palacios, M., Duggan, B., Shostak, I., Barry, M., Geskes, T., Wilkins, J., et al. (2011). Granzyme B inhibits vaccinia virus production through proteolytic cleavage of eukaryotic initiation factor 4 gamma 3. PLoS Pathog. 7:e1002447. doi: 10.1371/journal.ppat.1002447

Pando-Robles, V., Oses-Prieto, J., Rodríguez-Gandarilla, M., Meneses-Romero, E., Burlingame, A. L., and Batista, C. (2014). Quantitative proteomic analysis of Huh-7 cells infected with Dengue virus by label-free LC-MS. J. Proteomics 111, 16-29. doi: 10.1016/j.jprot.2014.06.029

Parker, J., Broering, T., Kim, J., Higgins, D., and Nibert, M. (2002). Reovirus core protein mu2 determines the filamentous morphology of viral inclusion bodies by interacting with and stabilizing microtubules. J. Virol. 76, 4483-4496. doi: 10.1128/JVI.76.9.4483-4496.2002

Pulit-Penaloza, J., Scherbik, S., and Brinton, M. (2012). Activation of Oas1a gene expression by type I IFN requires both STAT1 and STAT2 while only STAT2 is required for Oas1b activation. Virology 425, 71-81. doi: 10.1016/j.virol.2011.11.025

Qian, X., Hu, J., Zhao, J., and Chen, H. (2015). ATP citrate lyase expression is associated with advanced stage and prognosis in gastric adenocarcinoma. Int. J. Clin. Exp. Med. 8, 7855-7860.

Radtke, K., Dohner, K., and Sodeik, B. (2006). Viral interactions with the cytoskeleton: a hitchhiker's guide to the cell. Cell Microbiol. 8, 387-400. doi: 10.1111/j.1462-5822.2005.00679.x

Su, J., Li, S., Hu, X., Yu, X., Wang, Y., Liu, P., et al. (2011). Duck egg-drop syndrome caused by BYD virus, a new Tembusu-related flavivirus. PLoS ONE 6:e18106. doi: 10.1371/journal.pone.0018106

Sun, L., Li, Y., Zhang, Y., Han, Z., Xu, Y., Kong, X., et al. (2014). Adaptation and attenuation of duck tembusu virus strain $\mathrm{du} / \mathrm{ch} / \mathrm{lsd} / 110128$ following serial passage in chicken embryos. Clin. Vaccine Immunol. 21, 1046-1053. doi: 10.1128/CVI.00154-14

Svitkin, Y., Gradi, A., Imataka, H., Morino, S., and Sonenberg, N. (1999). Eukaryotic initiation factor 4GII (eIF4GII), but not eIF4GI, cleavage correlates with inhibition of host cell protein synthesis after human rhinovirus infection. J. Virol. 73, 3467-3472.

Wu, X., Wang, H., Bai, L., Yu, Y., Sun, Z., Yan, Y., et al. (2013). Mitochondrial proteomic analysis of human host cells infected with H3N2 swine influenza virus. J. Proteomics 91, 136-150. doi: 10.1016/j.jprot.2013.06.037
Xiao, H., Xu, L., Yamada, Y., and Liu, D. (2008). Coronavirus spike protein inhibits host cell translation by interaction with eIF3f. PLoS ONE 3:1494. doi: 10.1371/journal.pone.0001494

Yamamoto, Y., and Gaynor, R. (2004). I $\kappa$ B kinases: key regulators of the NF- $\kappa$ B pathway. Trends Biochem. Sci. 29, 72-79. doi: 10.1016/j.tibs.2003.12.003

Yu, K., Sheng, Z., Huang, B., Ma, X., Li, Y., Yuan, X., et al. (2013). Structural, antigenic, and evolutionary characterizations of the envelope protein of newly emerging duck tembusu virus. PLoS ONE 8: e71319. doi: 10.1371/journal.pone.0071319

Zhang, B., Liu, X., Chen, W., and Chen, L. (2013). IFIT5 potentiates anti-viral response through enhancing innate immune signaling pathways. Acta Biochim. Biophys. Sin. 45, 867-874. doi: 10.1093/abbs/gmt088

Zhang, X., and Wang, Y. (2015). Cell cycle regulation of VCIP135 deubiquitinase activity and function in p97/p47-mediated Golgi reassembly. Mol. Biol. Cell 26, 2242-2251. doi: 10.1091/mbc.E15-01-0041

Zheng, J., Sugrue, R., and Tang, K. (2011). Mass spectrometry based proteomic studies on viruses and hosts. Anal. Chim. Acta 702, 149-159. doi: 10.1016/j.aca.2011.06.045

Zheng, S., Li, Y., Zhang, Y., Li, X., and Tang, H. (2011). MiR-101 regulates HSV-1 replication by targeting ATP5B. Antiviral Res. 89, 219-226. doi: 10.1016/j.antiviral.2011.01.008

Zheng, X., Hong, L., Shi, L., Guo, J., Sun, Z., and Zhou, J. (2008). Proteomics analysis of host cells infected with infectious bursal disease virus. Mol. Cell. Proteomics 7, 612-625. doi: 10.1074/mcp.M700396-MCP200

Zhou, X., Michal, J., Zhang, L., Ding, B., Lunney, J., Liu, B., et al. (2013). Interferon induced IFIT family genes in host antiviral defense. Int. J. Biol. Sci. 9, 200-208. doi: 10.7150/ijbs.5613

Zhu, J., Zhang, Y., Ghosh, A., Cuevas, R., Forero, A., Dhar, J., et al. (2014). Antiviral activity of human oligoadenylate synthetases-like (OASL) is mediated by enhancing retinoic acid-inducible gene I (RIG-I) signaling. Immunity 40, 936-948. doi: 10.1016/j.immuni.2014.05.007

Conflict of Interest Statement: The authors declare that the research was conducted in the absence of any commercial or financial relationships that could be construed as a potential conflict of interest.

Copyright (c) 2016 Han, Zhao, Liu, Liu, Huang, Yang, An and Li. This is an openaccess article distributed under the terms of the Creative Commons Attribution License (CC BY). The use, distribution or reproduction in other forums is permitted, provided the original author(s) or licensor are credited and that the original publication in this journal is cited, in accordance with accepted academic practice. No use, distribution or reproduction is permitted which does not comply with these terms. 